\title{
The effect of hematocrit on the efficacy of phototherapy for neonatal jaundice
}

\author{
Angelo A. Lamola', Vinod K. Bhutani', Ronald J. Wong', David K. Stevenson' and Antony F. McDonagh ${ }^{1,2}$
}

BACKGROUND: The therapeutic phototherapy action spectrum ranges from 420 to $500 \mathrm{~nm}$. However, a recent report of improved efficacy of fluorescent "turquoise" light ( $490 \mathrm{~nm})$ as compared with blue light $(\sim 450 \mathrm{~nm})$ underscores the need to define an optimal action spectrum for precision-targeted phototherapy using very narrow wavelength ranges.

METHODS: We used a current semi-empirical model of the optical properties of skin for robust calculations of the fraction of light absorbed by bilirubin at various wavelengths that could be confounded by hemoglobin ( $\mathrm{Hb})$, melanin, and skin thickness. Applying assumptions regarding the wavelength dependence of bilirubin photochemistry, "action spectra" were assembled from the calculated values.

RESULTS: All the calculated action spectra displayed a peak between 472 and $480 \mathrm{~nm}$ (most at $476 \mathrm{~nm}$ ), which is a significant shift from the well-reported $460 \mathrm{~nm}$ absorption peak of bilirubin. Of note, the relative amplitudes of the action spectra showed an inverse relationship with hematocrit (Hct).

CONCLUSION: We speculate that a narrow range of light at $476 \mathrm{~nm}$ would be $60 \%$ more effective than blue (broadband) fluorescent lamps. Because $\mathrm{Hb}$ serves as a major competitor of bilirubin for light absorption, the calculations also predict that the efficacy of phototherapy is dependent on the Hct. A high Hct could reduce therapeutic efficiency.

A $\mathrm{n}$ in vivo model to better define the optimal wavelength range for precision-targeted phototherapy has been elusive. Advances in understanding of skin optical properties have provided an optical model of neonatal skin in the therapeutic range $(400-520 \mathrm{~nm})$ that allows facile calculation of the influences of skin structure, melanin pigmentation, and cutaneous hemoglobin $(\mathrm{Hb})$ on the action spectrum for phototherapy. Of these, $\mathrm{Hb}$ absorbs most of the visible light in the skin $(1,2)$. As a result, competition from $\mathrm{Hb}$ with bilirubin for light should strongly affect the wavelength region of light that is most effective for phototherapy of neonatal jaundice. Although $\mathrm{Hb}$ competition has been recognized and used, in part, to predict the action spectrum for phototherapy $(3,4)$, explicit reference to $\mathrm{Hb}$ competition appears to be absent from the recent literature. The reports of clinical studies showing that blue-green or green light may be more effective than blue light $(5,6)$ generally rationalize these observations on the general notion that the longer wavelengths "penetrate deeper into the skin" without specific reference to $\mathrm{Hb}$ absorbance.

Furthermore, significant competition for light by skin $\mathrm{Hb}$ also implies that the hematocrit (Hct) may in turn affect the efficacy of phototherapy. This possibility was recognized in 1974 by Lucey and Hewitt (7), who conjectured that the Hct "may be a contributing factor to the differences in response of serum bilirubin to light that one observes in different infants." They observed in in vitro irradiation studies that changes in the Hct affect diazo-reactive bilirubin disappearance in blood. Almost a decade elapsed before a clinical study (8) showed a modest decrease in phototherapy efficacy with an increase in Hct, and this was deemed statistically insignificant on the basis of limited data. No further investigations of the question have appeared since then.

To try to help explain these observations, we applied semiempirical calculations based on a modern and well-accepted model of skin optics $(1,2,9-14)$, known spectral data, and data on the blood and bilirubin levels in the skin, to show that Hct may, in fact, significantly affect phototherapy efficacy. In addition, the calculations allowed us to predict that a narrow wavelength-band emitting light source, such as light-emitting diodes (LEDs), at $476 \mathrm{~nm}$ would be the most efficient for phototherapy and would also minimize heating of the neonate by absorbed light. Previous observations $(5,15,16)$ that the optimal wavelength range for phototherapy is at longer wavelengths than the maximum of the bilirubin absorption spectrum $(460 \mathrm{~nm})$ are strongly supported by these calculations.

\section{RESULTS}

\section{Action Spectra}

Cutaneous bilirubin exists in the blood and in the extravascular compartment. To estimate the amount of extravascular bilirubin (for bilirubin levels that do not exceed the albuminbinding capacity), the ratio of extravascular to blood bilirubin is taken to be 1.5 , the same as the estimated ratio of extravascular to blood albumin (17). This assumes equilibration of bilirubin between the two albumin pools (17). Figure 1 shows the absorbance spectra of $\mathrm{Hb}$ and bilirubin in the molar ratio at $50 \% \mathrm{Hct}(2.6 \mathrm{mmol} / \mathrm{l} \mathrm{Hb}), 75 \%$ oxygenation, $15-\mathrm{mg} / \mathrm{dl}$ serum

'Division of Neonatal and Developmental Medicine, Department of Pediatrics, Stanford University School of Medicine, Stanford, California; ${ }^{2}$ Department of Medicine, Liver Center, University of California, San Francisco, California. Correspondence: Angelo A. Lamola (aalamola@stanford.edu)

Received 18 July 2012; accepted 8 January 2013; advance online publication 5 June 2013. doi:10.1038/pr.2013.67 


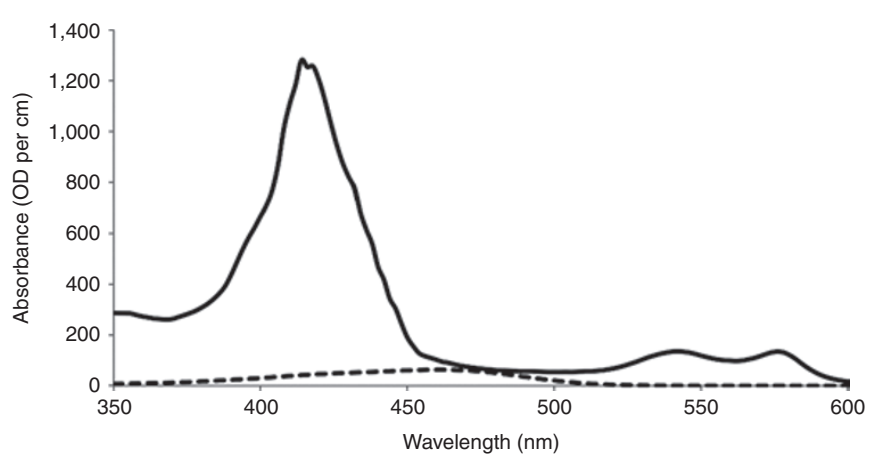

Figure 1. The absorbance of hemoglobin ( $\mathrm{Hb}$, solid line) and bilirubin/ human serum albumin (bilirubin, dashed line) at the concentrations of $2.6 \mathrm{mmol} / \mathrm{l}$ and $0.325 \mathrm{mmol} / \mathrm{l}$, respectively, that represent a hematocrit of $50 \%$, a serum unconjugated bilirubin at $15 \mathrm{mg} / \mathrm{dl}$, and an extravascular/ vascular bilirubin ratio of 1.5 . The $\mathrm{Hb}$ is oxygenated to $75 \%$. The bilirubin/ albumin absorbance (dashed line) is magnified by a factor of four for purposes of visualization. OD, optical density.

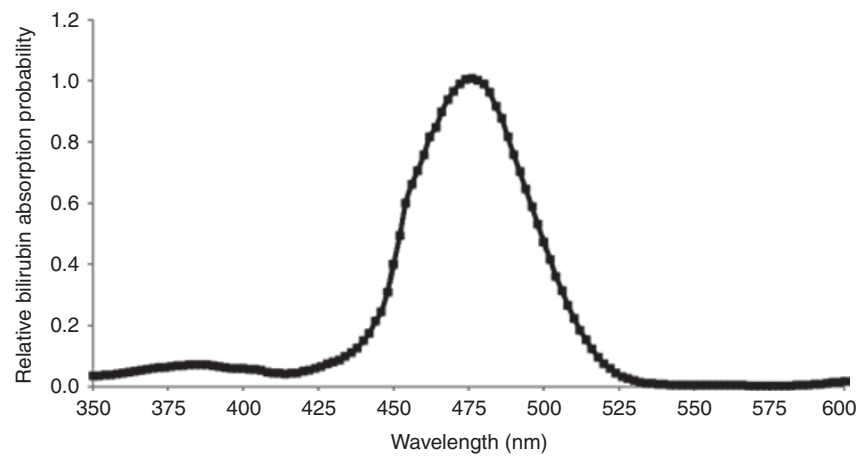

Figure 2. Calculated fraction of light absorbed by bilirubin $(15 \mathrm{mg} / \mathrm{dl}$ per serum volume) in blood, normalized to 1.0 at the maximum, for two hematocrit values, $30 \%$ (black squares) and $50 \%$ (solid line). The two curves are virtually identical and therefore appear as a single line. bilirubin $(0.13 \mathrm{mmol} / \mathrm{l}$ bilirubin in the blood), and an extravascular/vascular bilirubin ratio of 1.5 . It is clear that the $\mathrm{Hb}$ absorbance dominates that of bilirubin throughout all wavelengths and that the wavelength at which bilirubin would absorb the largest fraction (17\%) of incident light is $476 \mathrm{~nm}$. A plot of the fraction of light absorbed by bilirubin vs. wavelength is the first-order "action spectrum" and is shown (normalized to 1.0 at the maximum) in Figure 2.

Table 1 lists the maximum wavelengths ( $\lambda_{\max }$ values) at which the bilirubin absorbs the largest fraction of the light at varying Hct (20-70\%), oxygen saturation (75 and 90\%), and serum bilirubin $(5-25 \mathrm{mg} / \mathrm{dl})$ levels. It is striking that the $\lambda_{\max }$ values are virtually insensitive to these variations over the ranges examined. The calculated $\lambda_{\max }$ for $15-\mathrm{mg} / \mathrm{dl}$ serum bilirubin and various Hcts assuming no extravascular bilirubin are all at $476 \pm 1 \mathrm{~nm}$. Over the range $0-1.5$ for the ratio of extravascular to vascular bilirubin, the $\lambda_{\max }$ values are also minimally altered. The shapes of the action spectra are also minimally different. As an example, Figure 2 shows that the bilirubin absorption spectra (first-order "action spectra") at Hct values of 30 and $50 \%$ (at a constant $15-\mathrm{mg} / \mathrm{dl}$ serum bilirubin and $75 \%$ oxygenation) are virtually identical when normalized to 1.0 at the $\lambda_{\max }$.

The fraction of incident light absorbed by bilirubin in blood does, of course, vary substantially with the Hct, bilirubin, and oxygen saturation (Table 1). For example, the fraction absorbed by bilirubin at the $\lambda_{\max }$ is 2 times higher (11 vs. 5.4\%) for a Hct of $40 \%$ as compared with one of $60 \%$ (serum bilirubin $15 \mathrm{mg} / \mathrm{dl} ; 75 \%$ oxygenation).

In addition, accounting for skin backscatter does not change the calculated $\lambda_{\max }$ but reduces the fraction of light absorbed by bilirubin at $\lambda_{\max }$ by $\sim 7 \%$ (Table 1 ). Inclusion of epidermal melanin at $10 \% \mathrm{v} / \mathrm{v}$ (an overestimate for neonates of dark skin ethnicity) also does not shift the calculated $\lambda_{\text {max }}$ (Figure 3). As

Table 1. Percentage of light absorbed by bilirubin at $\lambda_{\max }$ as a function of hematocrit, degree of hemoglobin oxygenation, and serum bilirubin

\begin{tabular}{|c|c|c|c|c|c|c|c|c|c|}
\hline \multirow{3}{*}{$\begin{array}{l}\text { Bilirubin } \\
\text { (mg/dl) }\end{array}$} & \multirow[b]{3}{*}{ Hct (\%) } & \multirow{3}{*}{$\begin{array}{l}\text { Oxygenation } \\
\text { (\%) }\end{array}$} & \multirow[b]{3}{*}{$\lambda_{\max }(\mathrm{nm})$} & \multicolumn{6}{|c|}{$\%$ Light absorbed by bilirubin at $\lambda_{\max }$} \\
\hline & & & & \multicolumn{2}{|c|}{ First order } & \multicolumn{2}{|c|}{ With scatter } & \multicolumn{2}{|c|}{ Scatter + melanin } \\
\hline & & & & $r=0$ & $r=1.5$ & $r=0$ & $r=1.5$ & $r=0$ & $r=1.5$ \\
\hline 15 & 20 & 75 & 476 & 25.1 & 48.4 & 23.3 & 45.0 & 19.3 & 37.3 \\
\hline 15 & 30 & 75 & 476 & 15.6 & 32.5 & 14.5 & 30.3 & 12.0 & 25.0 \\
\hline 15 & 40 & 75 & 476 & 11.0 & 24.9 & 10.2 & 23.2 & 8.5 & 19.2 \\
\hline 15 & 50 & 75 & 476 & 7.7 & 17.3 & 7.2 & 16.1 & 6.0 & 13.3 \\
\hline 15 & 60 & 75 & 476 & 5.4 & 12.6 & 5.0 & 11.7 & 4.2 & 6.2 \\
\hline 15 & 70 & 75 & 476 & 3.6 & 7.6 & 3.3 & 7.1 & 2.8 & 5.9 \\
\hline \multicolumn{10}{|c|}{ Examples of variations in bilirubin, Hct, and oxygenation } \\
\hline 5 & 50 & 75 & 475 & 2.7 & 6.1 & 2.5 & 5.7 & 2.1 & 4.7 \\
\hline 10 & 50 & 75 & 476 & 5.3 & 11.9 & 4.9 & 11.0 & 4.2 & 8.7 \\
\hline 10 & 70 & 90 & 478 & 2.4 & 5.1 & 2.2 & 4.7 & 1.8 & 9.2 \\
\hline 15 & 50 & 90 & 476 & 7.2 & 16.2 & 6.7 & 15.1 & 5.6 & 12.5 \\
\hline 15 & 50 & 60 & 476 & 8.3 & 18.6 & 7.7 & 17.3 & 6.9 & 14.4 \\
\hline 20 & 50 & 75 & 476 & 10.1 & 22.7 & 9.4 & 21.1 & 7.8 & 17.5 \\
\hline 20 & 30 & 90 & 475 & 18.5 & 38.6 & 17.2 & 35.9 & 14.2 & 29.8 \\
\hline 25 & 50 & 75 & 475 & 20.3 & 45.7 & 18.9 & 42.5 & 15.7 & 33.2 \\
\hline
\end{tabular}

The wavelength $\left(\lambda_{\max }\right)$ at which the highest calculated fraction of incident light is absorbed by bilirubin for various values of hematocrit (Hct), degree of hemoglobin ( $\mathrm{Hb}$ ) oxygenation, serum bilirubin, and extravascular/vascular bilirubin ratios ( $r$ ) of 0 and 1.5 . 
expected, the presence of melanin does, of course, reduce the fraction of light absorption by bilirubin, by an additional 15\%.

When the wavelength dependence of the quantum yields for formation of lumirubin and $Z, E$-bilirubin are used to modify the action spectrum, the $\lambda_{\max }$ values shift to $480 \mathrm{~nm}$ (Figure 3 ) and $472 \mathrm{~nm}$ (not shown), respectively.

\section{Calculated Relative Efficacy of Light Sources}

The value of fraction of light absorption by bilirubin given in Table 1 relates to the efficacy of a very narrow bandwidth source $(1-2 \mathrm{~nm})$, such as a laser, to affect bilirubin photochemistry. To calculate the relative efficacy of a broader-band light source, the spectrum of the source and the action spectrum are convoluted (values at each wavelength multiplied together), and the results integrated over the wavelength range of the source



Figure 3. Calculated fraction of the light absorbed by bilirubin $(15 \mathrm{mg} / \mathrm{dl}$ per serum volume, hematocrit $50 \%$, and extravascular/vascular bilirubin ratio $=1.5$ ) normalized to 1.0 at the maximum. The solid curve included the wavelength dependence of the skin light backscatter, and the curve defined by the black squares included the wavelength dependence of melanin absorbance. The two curves are virtually identical. The dashed curve includes the wavelength dependence of the quantum yield for lumirubin production.

Table 2. Calculated relative photon absorption rates of bilirubin for various light sources

\begin{tabular}{|c|c|}
\hline Source & $\begin{array}{c}\text { Relative } \\
\text { bilirubin } \\
\text { absorption } \\
\text { rate }\end{array}$ \\
\hline \multicolumn{2}{|l|}{ LEDs-neoBLUE-type spectral distribution centered at } \\
\hline $450 \mathrm{~nm}$ & 0.62 \\
\hline $460 \mathrm{~nm}$ & 0.85 \\
\hline $470 \mathrm{~nm}$ & 0.96 \\
\hline $476 \mathrm{~nm}$ & $1.0^{\mathrm{a}}$ \\
\hline $480 \mathrm{~nm}$ & 0.98 \\
\hline $490 \mathrm{~nm}$ & 0.79 \\
\hline Philips TL20W/52 blue lamp $\left(\lambda_{\max }=450 \mathrm{~nm}\right)$ & 0.61 \\
\hline OSRAM L18W/860"turquoise"lamp ( $\left.\lambda_{\max }=490 \mathrm{~nm}\right)$ & 0.72 \\
\hline \multicolumn{2}{|c|}{$\begin{array}{l}\text { Relative photon absorption rates of bilirubin for various light sources (Philips, } \\
\text { Amsterdam, The Netherlands; OSRAM, Munich, Germany) were calculated using the } \\
\text { calculated action spectrum represented by the solid line in Figure } \mathbf{3} \text {. }\end{array}$} \\
\hline \multicolumn{2}{|c|}{$\begin{array}{l}\text { aThe results pertain to equalized total irradiances of the light sources and are referenced } \\
\text { to the } 476 \mathrm{~nm} \text { light-emitting diode (LED) source set to 1.0. }\end{array}$} \\
\hline
\end{tabular}

spectrum. Table 2 gives the results of the convolutions/integrations for LEDs and fluorescent light with the action spectra calculated for $50 \% \mathrm{Hct}, 75 \%$ oxygenation, $15-\mathrm{mg} / \mathrm{dl}$ serum bilirubin, and inclusion of backscatter. The source spectra were normalized to equal total irradiances. The results are the same for any ratio of extravascular to vascular bilirubin from 0 to 1.5 because the action spectra are virtually identical over this ratio range (data not shown).

\section{DISCUSSION}

\section{Model Justification}

Accounting for both light backscatter and melanin as a transmission filter in our model is well founded $(9,12,14)$. Open to discussion is the assumption that all the light transmitted into the dermis and not scattered back out is absorbed by $\mathrm{Hb}$ and bilirubin in the dermis and not much further into subdermal tissue. The strong light-absorbing power of $\mathrm{Hb}$ is indicated by the fact that, in the range of 400 to $440 \mathrm{~nm}$ or so, a stack of only two to four red cells has an absorbance of at least 1.0 (more than $90 \%$ of the light is absorbed). At higher wavelengths (440$600 \mathrm{~nm}$ ), the absorbance of the $\mathrm{Hb}$ is reduced but remains sufficiently high to justify the model. The blood content of the skin of 47 neonates, measured by Jacques et al. (9), ranged from 0.8 to $2.4 \%$ of the volume (4-12 $\mathrm{mg}$ of $\mathrm{Hb}$ per gram). Therefore, for a 0.5 - to 1.5 -mm-thick dermis, blood content of $1.5 \%(\mathrm{v} / \mathrm{v})($ at a Hct of $50 \%$ ) is sufficient to absorb at least $30 \%$ of light in the range of $440-600 \mathrm{~nm}$. This takes into account that the light entering the dermis undergoes efficient diffusive scatter such that the effective path length is significantly larger than the straight path. Given the even greater blood content of subdermal tissue, it is then expected that almost all the light in this range would be absorbed either within the dermis or within the dermis and another 1-2 mm below the dermis.

Because light within the skin is diffuse, it is also valid, for the present purpose, to model the distribution of blood and bilirubin in the skin as homogeneous $(9,12)$. Demonstrated by fluorescence studies, the distribution of bilirubin with respect to $\mathrm{Hb}$ in blood can also be considered homogeneous (18). The impinging light from phototherapy sources is uncollimated and thus helps ensure the extensive diffusion of light within the skin.

Other points for discussion concern the presence of extravascular bilirubin in the skin and its importance to the phototherapy of bilirubin. There is much evidence that until the bilirubin exceeds its binding sites on albumin, most of the bilirubin remains bound to albumin (19). That this is valid in vivo is indicated by the measurement of bilirubin in the skin by transcutaneous reflectometry, which tracks the serum bilirubin level in the blood $(12,17,20,21)$. There is no evidence that extravascular bilirubin binds to other skin components (17). Blood flow through the skin is substantial so that a continuous new supply of blood is available for exposure to phototherapy light. By contrast, the transport of bilirubin out of and back into the vasculature is comparatively slow. The observation that photoisomers of bilirubin appear rapidly in the blood on commencement of phototherapy, more rapidly than is thought 
to accommodate transport from outside the vasculature, indicates that the blood compartment is an important site with regard to phototherapy (22).The "rebound" in serum bilirubin sometimes observed after cessation of phototherapy generally reflects continued bilirubin production due to hemolysis and not re-entrance into the blood from extravascular sites $(23,24)$.

How would the presence of bilirubin outside the vasculature complicate the model used here? Assuming a minor role in the phototherapy per se, such bilirubin would act solely as an additional filter absorbing otherwise usable light, thus reducing overall efficacy. This "self-filtering" would also shift the maximum of the light absorbed by the blood bilirubin slightly to longer wavelengths. The mixture of photoproducts from extravascular bilirubin could be different from that for the blood bilirubin for several reasons, including the higher probability that more than one photon might be absorbed by extravascular bilirubin molecules. The kinetics of photoproduct clearance could lead to complex kinetics of observed serum bilirubin changes.

\section{Action Spectra}

It is evident from Figure 1 that $\mathrm{Hb}$ in blood should overwhelmingly compete with bilirubin for light absorption and that this competition is stronger from 400 to $460 \mathrm{~nm}$ than it is from 460 to $500 \mathrm{~nm}$. The most favorable range for bilirubin to compete for light absorption is 475 to $480 \mathrm{~nm}$. Moreover, the fraction of light absorbed by the bilirubin as a function of wavelength, the "action spectrum," should peak in that range, at least $15 \mathrm{~nm}$ higher than the peak $(460 \mathrm{~nm})$ of the bilirubin/ human serum albumin absorption spectrum. The calculated wavelengths of the maximum fractional absorption for bilirubin for various values of Hct, degree of oxygenation, inclusion of scatter and melanin, and change in ratio of extravascular to vascular bilirubin of 0 to 1.5 , given in Table 1 , all fall within the range $475-478 \mathrm{~nm}$. The shapes of the spectra are also nearly the same (Figures 2 and 3 ).

Bilirubin undergoes various photochemical transformations $(15,16,25)$. The two main transformations are configurational isomerization (to $Z, E$-bilirubin) and structural isomerization (to lumirubin). It is suggested that the rapidly excreted lumirubin is a key product regards phototherapy $(16,26)$. Both the formation of $Z, E$-bilirubin and lumirubin exhibit wavelengthdependent yields. Using reported $(16,25)$ wavelength dependencies to modify the "action spectra" accounts for the efficiency of the photochemistry. When the yield of lumirubin is included, $\lambda_{\max }$ shifts to $480 \mathrm{~nm}$ (Figure 3). When the yield of $Z$,E-bilirubin is included, $\lambda_{\text {max }}$ shifts to $472 \mathrm{~nm}$ (data not shown). That is, the "corrections" for the wavelength dependence of the photochemistry shift the $\lambda_{\max }$ by only $\pm 4 \mathrm{~nm}$.

A persistent finding of the present model is that competition for phototherapy light by $\mathrm{Hb}$ dominates to the extent that changes in the ratio of $\mathrm{Hb}$ to bilirubin in the clinical range, the inclusion or not of extravascular bilirubin, the inclusion or not of skin scatter or a generous amount of epidermal melanin, and the inclusion of the wavelength dependence of bilirubin photochemistry do not greatly alter the $\lambda_{\max }$ values or shapes of the "action spectra." The total range of calculated $\lambda_{\max }$ values, from 472 to $480 \mathrm{~nm}$, represents significantly longer wavelengths than the currently recommended range of $450 \pm 20 \mathrm{~nm}$ $(21,27)$ and is consistent with previous predictions $(3,4,15,16)$.

Previous predictions of action spectra based on calculations $(3,4)$ did not appropriately take into account the skin $\mathrm{Hb}$. The skin optics model used was an early, incomplete, model (28) that considered that all the bilirubin is in a layer below skin layers containing $\mathrm{Hb}$ and melanin. Some optical parameters and skin blood content were also based on early data, and there was no inclusion of extravascular bilirubin. The calculations were based on a complex analytical approach, perhaps the reason why these reports have not had the influence they deserve. In fact, in an article published in 1984, Pratesi et al. (4) concluded that a narrow-band source peaked between 475 and $480 \mathrm{~nm}$ would be optimum, which is similar to the current findings. However, the model and simpler methods used here facilitate broad examination of the effects of variations in bilirubin level, Hct, oxygenation level, and so on, to provide a robust prediction of the optimum wavelength region for phototherapy.

\section{Effect of Hct on Required Irradiance}

Whereas the $\lambda_{\max }$ values of the calculated action spectra are minimally affected by the $\mathrm{Hb}$ to bilirubin ratio, the amount of incident light absorbed by bilirubin is significantly reduced as the amount of $\mathrm{Hb}$ increases (Table 1). The rate of light absorption by the bilirubin is related to the rate of bilirubin photochemistry and hence the efficacy of phototherapy. It must be pointed out that the results here pertain to the initial rate of bilirubin photochemistry. Other factors, including the kinetics of clearance of extravascular bilirubin, may affect the extent of bilirubin reduction measured after many hours of phototherapy.

The Hct of infants under phototherapy is not a parameter considered in clinical practice. However, the results listed in Table 1 may indicate a clinically significant consideration. Consider the "normal" range of Hcts in neonates, i.e., $40-60 \%$. According to Table 1, going from a Hct of $40 \%$ to $60 \%$ could mean a twofold reduction in absorption of light by bilirubin. Or, to put it the other way around, a twofold increase in irradiance or of duration would be required to achieve the same amount of light absorbed by bilirubin for an infant with a Hct of $60 \%$ vs. an infant with a Hct of $40 \%$. Of course, the infant with $60 \%$ Hct has only two-thirds of the total bilirubin burden (assuming equal blood volumes) and so the rate of diminution of serum bilirubin concentration, the usual measure of phototherapy efficacy, all else being equal, would be reduced by $33 \%$. The relative rates of absorption of light by bilirubin as a function of Hct are shown in Figure 4, normalized to 1 for $50 \%$ Hct. These rates are proportional to the quantity of bilirubin undergoing photochemical alteration per unit time. All else being equal, they represent the quantity of bilirubin (number of bilirubin molecules) being removed. For the same starting serum bilirubin concentration, the total bilirubin burden increases with lower Hct, and the relative rate of serum bilirubin decrement (shown in Figure 4, normalized to 50\% 


\section{Articles $\mid$ Lamola e tal.}

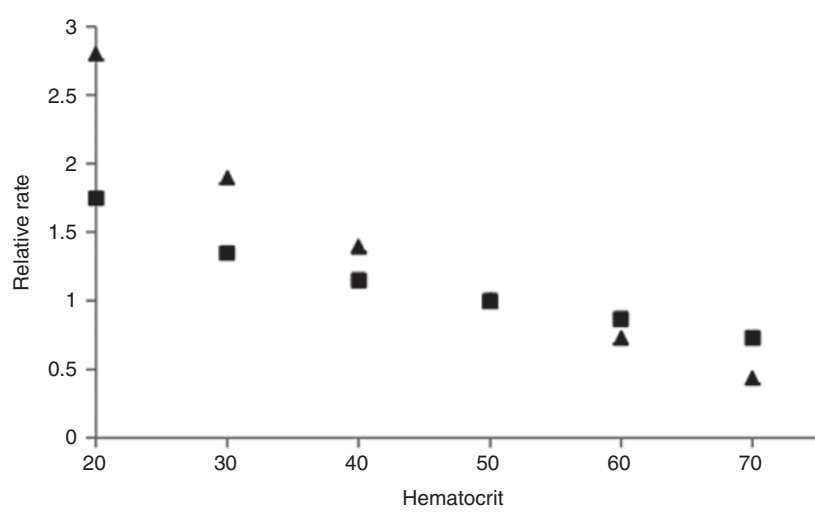

Figure 4. Relative calculated rates of light absorption by bilirubin (black triangles) and related relative rates for serum bilirubin decrement (black squares) as a function of hematocrit (Hct) normalized to 1 for $50 \% \mathrm{Hct}$, all other clinical parameters being equal.

Hct $=1)$ is moderated. In the only clinical study of the effect of Hct (8), an average of $7.5 \%$ reduction in the rate of serum bilirubin decrement at $24 \mathrm{~h}$ of therapy was observed with an increase of average Hct from 50 to $67 \%$, which was deemed statistically insignificant. The current model predicts a $28 \%$ reduction in the initial rate, all other clinical parameters being equal. Such a difference should be observable in an appropriately designed clinical study.

Clinical studies comparing the efficacy of phototherapy systems $(5,6,29,30)$ exhibit quite "noisy" data. There are many clinical parameters that may be associated with this variation. These include differences in bilirubin production rates, blood volume, photoproduct excretion rates, area exposed, and so on. In none of the studies was Hct controlled or taken into account in data interpretation. The results of the calculations here indicate that a portion of the variability in clinical studies of phototherapy efficacy may well have been due to variations in the Hcts of the infants. Of course, both Hct and blood volume are constantly changing parameters in some infants.

\section{Comparison of Light Sources}

The results (Table 2) of convolutions of the spectra of various light sources with the action spectrum calculated for Hct $50 \%$, $75 \%$ oxygenation, $15-\mathrm{mg} / \mathrm{dl}$ serum bilirubin, and including light scatter and melanin provide interesting considerations. First, it is clear that, for equal total irradiance, the differences in calculated phototherapy efficacy among the sources, at most $\sim 50 \%$, can be made up by concomitant changes in the irradiance and/or therapy time. That is, all the light sources considered here can be clinically useful. However, the most efficient source would be a bank of narrow-band LEDs with peak emission at $476 \mathrm{~nm}$. Such a source would be 1.5 times more effective than the blue Philips lamp (Philips, Amsterdam, The Netherlands), for equal irradiance, and avoids lower-wavelength blue light that is less useful for therapy because of an additional heat burden.

The calculated difference in efficacy between the Philips blue and OSRAM (Munich, Germany) "turquoise" lamp, 18\%, is very close to the average difference found in a recent clinical study (5). The conclusions of that study and other studies have shown that for equal irradiance, blue-green light may be more effective than blue light. (However, in comparing light sources, clinicians are reminded that they should neither rely on visual comparisons nor manufacturers' descriptions of color and should examine the actual spectra of the sources.)

\section{Heat Deposition via Absorbed Light}

The term "heat deposition" here refers to the deposition of heat due to light absorption within the skin and not to other modes of heat transfer from the phototherapy device. Light absorbed by either $\mathrm{Hb}$ or bilirubin is converted into heat within nanoseconds, first localized in the molecules themselves and then quickly transferred to their surroundings. The use of the idealized $(476 \mathrm{~nm})$ LED source with the recommended $(21,27)$ average irradiance of $30 \mu \mathrm{W} / \mathrm{cm}^{2} / \mathrm{nm}$ over an irradiated area of $1,500 \mathrm{~cm}^{2}$ would generate heat into the dermal/subdermal vasculature and tissue of the infant at a rate of $\sim 2 \mathrm{~W}$, or $\sim 1,700$ calories per h. If Philips Tl20W/52 lamps are used at an irradiance to obtain the same therapeutic efficacy as the $476 \mathrm{~nm} \mathrm{LED}$ source, then heat would be deposited at $3.3 \mathrm{~W}$.

\section{Conclusions}

Calculations based on this semi-empirical model show that $\mathrm{Hb}$ in the skin is the major competitor of bilirubin for absorption of light used in neonatal jaundice phototherapy. This competition is strongest from 400 to $460 \mathrm{~nm}$ and shifts the maximum of the "action spectrum" for phototherapy to 475 to $480 \mathrm{~nm}$. A high Hct will reduce therapeutic efficacy, a prediction that needs clinical verification. The calculations predict that a narrow-band LED source at $476 \mathrm{~nm}$ would be more efficient than broadband fluorescent lamps, maximizing therapeutic efficacy and reducing the heat load due to absorption of less effective light.

A recent study suggests an increased mortality among verylow-birth weight infants under aggressive phototherapy (31). If aggressive phototherapy entails a higher dose of lower-wavelength blue light exposure, in the context of low Hct, making more light available to interact with other blue light-absorbing entities, the potential for aberrant reactions to occur (e.g., photosensitized oxidative damage (31-34) or alterations of riboflavin $(35,36))$ might be higher. Unfortunately, light sources and light doses were not controlled, and the Hcts were not reported in that study.

\section{METHODS}

\section{Model and Calculations}

Skin optics. Enabled by advances in physical optics and electronics, the optical properties of human skin have become well described $(1,2,9-14)$. This has resulted in a model of skin optics that has led to improvements in devices such as transcutaneous bilirubinometers $(2,9,14,21,37)$. Those features of this model for skin optics germane to the spectral region (400-520 nm) prescribed for jaundice therapy are reviewed here. In this region, only a small fraction $(\sim 8 \%)$ of the light impinging on the skin is backscattered $(2,12,27,28)$. The wavelength dependence of backscattering from the skin is mild, otherwise nonpigmented skin would appear distinctly bluish rather than white. The 


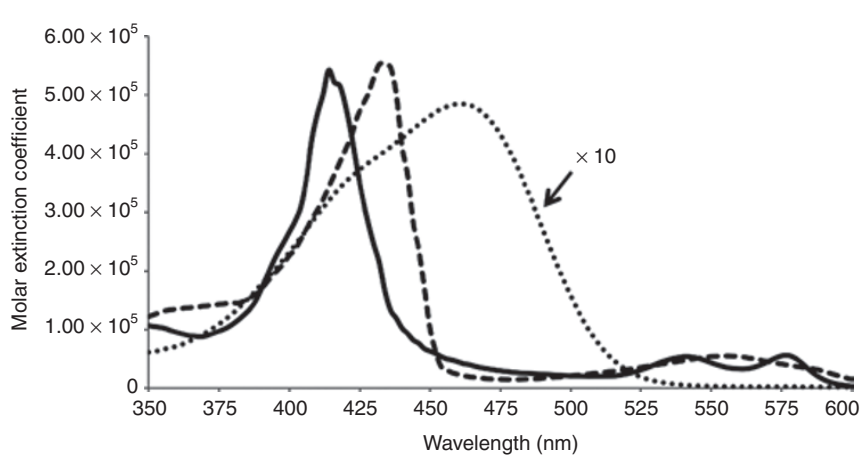

Figure 5. Absorption spectra of oxyhemoglobin $\left(\mathrm{O}_{2} \mathrm{Hb}\right.$, solid line), deoxyhemoglobin ( $\mathrm{HHb}$, dashed line), and bilirubin bound to human serum albumin (bilirubin/Alb, dotted line). The bilirubin absorption curve (dotted line) is magnified by a factor of 10 ; its extinction coefficient at the maximum $(460 \mathrm{~nm})$ is $48,400 \mathrm{~cm}^{-1} \mathrm{~mol} / \mathrm{l}^{-1}$.

remaining fraction is diffusely scattered within the dermis and deeper tissue unless and until it is absorbed by pigments there. Backscatter from the epidermis is small, and this skin layer can be modeled as a transmission filter to account for any melanin present $(9,12)$.

It is well established that $\mathrm{Hb}$ is the major absorber of visible light in the dermis, especially in the regions $400-425 \mathrm{~nm}$ and 500$600 \mathrm{~nm}(1,2,9,12,14)$. Other pigments whose light absorbance can be observed when their levels are elevated include bilirubin and carotenoids. Other endogenous pigments are present at much lower concentrations.

The model. The model pertains to light of wavelength $400-520 \mathrm{~nm}$. The wavelength dependence and magnitude of light scattered back out of the skin are modeled as a transmission filter in the epidermis. The absorption due to melanin is treated likewise. The remaining light, diffusively scattered, is considered to be eventually absorbed entirely by $\mathrm{Hb}$ and bilirubin within the dermis or within the dermis and not very much deeper in subdermal tissue. Because the light is diffuse, from an optics perspective, both the bilirubin and $\mathrm{Hb}$ are considered homogeneously distributed. The latter two aspects of the model provide for simplification of the calculations. The kinds of ray tracing and mathematical approach necessary for analysis in reflectance methods for bilirubin assays $(9,12)$ are not necessary for estimating the fractional absorption of light by the bilirubin in the skin.

Spectra. Utilized here is a tabulation of the extinction coefficients $\left(\mathrm{cm}^{-1} \mathrm{~mol} / \mathrm{l}^{-1}\right)$ for oxy- and deoxyhemoglobin at every $2 \mathrm{~nm}$ compiled by Prahl (38). Plots of these spectra are shown in Figure 5. The small differences between the spectra of adult and fetal Hbs (39) are ignored. A spectrum of purified $(4 Z, 15 Z)$-bilirubin IX $\alpha$ in the presence of excess human serum albumin was provided by A.F.M. (Figure 5). Although there is variation in the absorbance values reported for melanin in skin, there is agreement on the absorption spectrum (not shown), which is a falling curve, nearly linear, between 400 and $520 \mathrm{~nm}$. A spectrum reported by Kollias (40) was used. The wavelength dependence (not shown) of skin backscatter given by Jacques et al. $(9,12)$ was employed. The spectra of the light sources expressed as irradiance $\left(\mu \mathrm{W} / \mathrm{cm}^{2} / \mathrm{nm}\right)$ as a function of wavelength were obtained from the literature (5) and from websites of the manufacturers.

Impact of quantity and concentration. Although the amount of extravascular albumin in a volume of skin may be as much as 1.5 times that in the blood compartment of the same volume, the ratio of concentrations of extravascular albumin to vascular albumin is of the order of 0.02 because in newborn skin the vascular volume is of the order of $1.5 \%$ of the skin (9). If the ratio of extravascular bilirubin to blood bilirubin is also 1.5 , then for a serum bilirubin of $15 \mathrm{mg} / \mathrm{dl}$ $(0.26 \mathrm{mmol} / \mathrm{l})$ and a Hct of $50 \%$, the blood bilirubin concentration is $0.13 \mathrm{mmol} / \mathrm{l}$, the extravascular bilirubin concentration is $\sim 0.0026$ $\mathrm{mmol} / \mathrm{l}$, and the average concentration of bilirubin in the skin volume is $\sim 0.005 \mathrm{mmol} / \mathrm{l}$. For a Hct of $50 \%$, the $\mathrm{Hb}$ concentration in the blood is $2.6 \mathrm{mmol} / \mathrm{l}$ and the concentration referenced to the skin volume is $0.04 \mathrm{mmol} / \mathrm{l}$, giving a bilirubin/Hb molar ratio of 0.125 .

Calculations. Calculations were performed numerically using the spreadsheet methodology. Spectra were tabulated at 2-nm intervals.

The absorption coefficients for any mixture of oxyhemoglobin and deoxyhemoglobin are simply the weighted combination of the individual values. The oxygenation level in the skin (average of arterial and venous oxygenation) used for most calculations was $75 \%$. Calculations were also performed for 90 and $60 \%$ oxygenation.

Under the assumption that practically all the light transmitted through the epidermis is absorbed by the combination of $\mathrm{Hb}$ and bilirubin (B), the fraction $\left(\mathrm{F}_{\mathrm{B}}\right)$ of that light absorbed by the bilirubin at a particular wavelength is closely given by:

$$
F_{\mathrm{B}}(\lambda)=\varepsilon_{\mathrm{B}} c_{\mathrm{B}} /\left(\varepsilon_{\mathrm{B}} c_{\mathrm{B}}+\varepsilon_{\mathrm{Hb}} c_{\mathrm{Hb}}\right),
$$

where $\varepsilon_{\mathrm{B}}$ is the molar extinction coefficient of bilirubin, $c_{\mathrm{B}}$ is the bilirubin concentration, $\varepsilon_{\mathrm{Hb}}$ is the weighted average extinction coefficient for the oxyhemoglobin/deoxyhemoglobin mixture, and $c_{\mathrm{Hb}}$ is the $\mathrm{Hb}$ concentration. The equation can be rearranged to:

$$
F_{\mathrm{B}}(\lambda)=\varepsilon_{\mathrm{B}} R /\left(\varepsilon_{\mathrm{B}} R+\varepsilon_{\mathrm{Hb}}\right)
$$

where $\mathrm{R}=c_{\mathrm{B}} / c_{\mathrm{Hb}}$, the molar ratio of the bilirubin to $\mathrm{Hb}$.

The plot of $F_{\mathrm{B}}$ vs. $\lambda$ calculated in this manner represents a firstorder action spectrum for phototherapy before correction for epidermal transmission and skin backscatter, corrections for wavelength dependences of relevant photochemical process(es), and any corrections for differences in the roles of vascular and extravascular bilirubin.

Using the wavelength dependence of backscatter for neonatal dermis (gestational age: $40 \mathrm{wk}$ ) given by Jacques et al. $(9,12)$, the fraction of the light that is not scattered back out was estimated for every $2 \mathrm{~nm}$. The reduced transmission through the epidermis due to melanin (at 10\% v/v) was estimated from absorption spectrum reported by Kollias (40).

The wavelength dependences of the quantum yields for the photochemical transformation of bilirubin to lumirubin and to $4 Z, 15 E$ bilirubin were taken from those reported by McDonagh et al. and Agati et al. $(16,25)$ and represent the most reliable values to date. A linear extrapolation was utilized to complete a table of yields for wavelengths between those reported.

Emission spectra of various blue LEDs with peak wavelengths in the $400-500 \mathrm{~nm}$ range are nearly symmetric around their peaks with bandwidths of 25-30 nm (21,41-43). The spectrum of the LEDs used here $(460 \mathrm{~nm}$ peak and $28 \mathrm{~nm}$ bandwidth) was from that reported from a commercially available and widely used phototherapy system $(41,42)$. The relative intensity at every $2 \mathrm{~nm}$ was incorporated into the database. Using the same spectral width, spectra peaked at 450,470 , 476,480 , and $490 \mathrm{~nm}$ were generated and incorporated into the database. Emission spectra for the blue Philips TL20W/52 and turquoise OSRAM L18W/860 lamps (5) were likewise entered into the database. Each of these spectra was normalized to the same relative total irradiance as the $460 \mathrm{~nm}$ LEDs. Then each spectrum was converted from relative irradiance to relative photon intensity (photon intensity is proportional to irradiance $\times$ wavelength).

To calculate the relative photon intensity absorbed by bilirubin for a light source and a particular action spectrum, the product of the fraction of the incident light absorbed by bilirubin, $F_{\mathrm{B}}(\lambda)$, times the relative photon intensity of the source was obtained for each recorded wavelength and then summed over the entire wavelength range. This relative photon absorption rate was then converted back to a relative rate based on total irradiance using the weighted average of the irradiance to photon intensity ratio for the source spectrum.

\section{ACKNOWLEDGMENTS}

A.A.L., V.K.B., R.J.W., and D.K.S. are sad to report that A.F.M. passed away before the final editing of this article. A.F.M. was a major contributor to 
bilirubin research over decades and, for this, was recently honored with the Landmark Award of the American Academy of Pediatrics. John F. Ennever and Thor W.R. Hansen are thanked for valuable discussions. Steven Jacques provided an education on skin optics and reviewed some of the calculations. Jenny Li produced the graphics.

\section{REFERENCES}

1. Jacques SL. Origins of tissue optical properties in the UVA, visible, and NIR regions. In: Afano RR, Fujimoto JG, eds. Advances in Optical Imaging and Photon Migration. Oregon: Optical Society of America TOPS Proceedings, 1996:374-69.

2. Nielson KP, Zhao L, Stamnes JJ, Stamnes K, Moan J. The optics of human skin: aspects important for human health. In: Bjertness E, ed. Solar Radiation and Human Health. Oslo: Norwegian Academy of Science and Letters, 2008:35-46.

3. Agati G, Fusi F, Donzelli GP, Pratesi R. Quantum yield and skin filtering effects on the formation rate of lumirubin. J Photochem Photobiol B, Biol 1993; 18:197-203.

4. Pratesi R, Ronchi L, Cecchi G, et al. Skin optics and phototherapy of jaundice. Photochem Photobiol 1984;40:77-83.

5. Ebbesen F, Madsen P, Støvring S, Hundborg H, Agati G. Therapeutic effect of turquoise versus blue light with equal irradiance in preterm infants with jaundice. Acta Paediatr 2007;96:837-41.

6. Seidman DS, Moise J, Ergaz Z, et al. A prospective randomized controlled study of phototherapy using blue and blue-green light-emitting devices, and conventional halogen-quartz phototherapy. J Perinatol 2003;23:123-7.

7. Lucey JF, Hewitt J. Recent observations on light and neonatal jaundice. In: Brown AK, Showacre J, eds. Phototherapy for Neonatal Jaundice DHEW Publication No (NIH). 1974:76-1075.

8. Granati B, Felice M, Fortunato A, Giancola G, Rubaltelli FF. Sites of action of light during phototherapy. Biol Neonate 1983;43:1-8.

9. Jacques SL, Saidi IS, Ladner A, Oelberg, D. Developing an optical fiber reflectance spectrometer to monitor bilirubinemia in neonates. Proc SPIE 1997;2975:115-24.

10. Jacques SL, Saidi IS, Tittel FK. Average depth of blood vessels in skin and lesions deduced by optical fiber spectroscopy. Proc SPIE 1994;2128:231-7.

11. Krishnaswamy A, Baranoski VG. A Study on Skin Optics. Canada: School of Computer Science University of Waterloo, 2004; report CS-2004-01.

12. Saidi IS, Jacques SL, Tittel FK. Mie and Rayleigh modeling of visible-light scattering in neonatal skin. Appl Opt 1995;34:7410-8.

13. Tuchin V. Tissue Optics-Light Scattering and Instruments for Medical Diagnosis, 2nd edn. Bellingham, WA: Society of Photographic Instrumentation Engineers, 2007.

14. Zonios G, Bykowski J, Kollias N. Skin melanin, hemoglobin, and light scattering properties can be quantitatively assessed in vivo using diffuse reflectance spectroscopy. J Invest Dermatol 2001;117:1452-7.

15. Ennever JF. Blue light, green light, white light, more light: treatment of neonatal jaundice. Clin Perinatol 1990;17:467-81.

16. McDonagh AF, Agati G, Fusi F, Pratesi R. Quantum yields for laser photocyclization of bilirubin in the presence of human serum albumin. Dependence of quantum yield on excitation wavelength. Photochem Photobiol 1989;50:305-19.

17. Knudsen A, Brodersen R. Skin colour and bilirubin in neonates. Arch Dis Child 1989;64:605-9.

18. Lamola AA, Eisinger J, Blumberg WE, Patel SC, Flores J. Flurorometric study of the partition of bilirubin among blood components: basis for rapid microassays of bilirubin and bilirubin binding capacity in whole blood. Anal Biochem 1979;100:25-42.

19. Ahlfors CE, Wennberg RP, Ostrow JD, Tiribelli C. Unbound (free) bilirubin: improving the paradigm for evaluating neonatal jaundice. Clin Chem 2009;55:1288-99.
20. Yamanouchi I, Yamauchi Y, Igarashi I. Transcutaneous bilirubinometry: preliminary studies of noninvasive transcutaneous bilirubin meter in the Okayama National Hospital. Pediatrics 1980;65:195-202.

21. Bhutani VK; Committee on Fetus and Newborn; American Academy of Pediatrics. Phototherapy to prevent severe neonatal hyperbilirubinemia in the newborn infant 35 or more weeks of gestation. Pediatrics 2011;128:e1046-52.

22. Mreihil K, McDonagh AF, Nakstad B, Hansen TW. Early isomerization of bilirubin in phototherapy of neonatal jaundice. Pediatr Res 2010;67:656-9.

23. Maisels MJ, Kring E. Rebound in serum bilirubin level following intensive phototherapy. Arch Pediatr Adolesc Med 2002;156:669-72.

24. Kaplan M, Kaplan E, Hammerman C, et al. Post-phototherapy neonatal bilirubin rebound: a potential cause of significant hyperbilirubinaemia. Arch Dis Child 2006;91:31-4.

25. Agati G, Fusi F, Pratesi R, McDonagh AF. Wavelength-dependent quantum yield for $\mathrm{Z}-\mathrm{E}$ isomerization of bilirubin complexed with human serum albumin. Photochem Photobiol 1992;55:185-90.

26. McDonagh AF, Lightner DA. Phototherapy and the photobiology of bilirubin. Semin Liver Dis 1988;8:272-83.

27. Bhutani VK, Cline BK, Donaldson KM, Vreman HJ. The need to implement effective phototherapy in resource-constrained settings. Semin Perinatol 2011;35:192-7.

28. Anderson RR, Parrish JA. The optics of human skin. J Invest Dermatol 1981;77:13-9.

29. Maisels MJ, Kring EA, DeRidder J. Randomized controlled trial of lightemitting diode phototherapy. J Perinatol 2007;27:565-7.

30. Morris BH, Tyson JE, Stevenson DK, et al. Efficacy of phototherapy devices and outcomes among extremely low birth weight infants: multi-center observational study. J Perinatol 2013;33:126-33.

31. Morris BH, Oh W, Tyson JE, et al.; NICHD Neonatal Research Network. Aggressive vs. conservative phototherapy for infants with extremely low birth weight. N Engl J Med 2008;359:1885-96.

32. Gathwala G, Sharma S. Phototherapy induces oxidative stress in premature neonates. Indian J Gastroenterol 2002;21:153-4.

33. Gathwala G, Sharma S. Oxidative stress, phototherapy and the neonate Indian J Pediatr 2000;67:805-8.

34. Aycicek A, Erel O. Total oxidant/antioxidant status in jaundiced newborns before and after phototherapy. J Pediatr (Rio J) 2007;83:319-22.

35. Gromisch DS, Lopez R, Cole HS, Cooperman JM. Light (phototherapy)induced riboflavin deficiency in the neonate. J Pediatr 1977;90:118-22.

36. Knobloch E, Hodr R, Houdková V. [Spectrofluorimetric determination of FAD, FMN and free riboflavin in the blood]. Cesk Farm 1988;37: 108-11.

37. Bosschaart N, Kok JH, Newsum AM, et al. Limitations and opportunities of transcutaneous bilirubin measurements. Pediatrics 2012;129:689-94.

38. Prahl S,Oregon Medical Laser Center. Tabulated molar extinction coefficient for hemoglobin in water. Oregon Medical Laser Center, 1999. (http:// omlc.ogi.edu/spectra/hemoglobin/summary.html.)

39. Zijlstra WG, Buursma A, Meeuwsen-van der Roest WP. Absorption spectra of human fetal and adult oxyhemoglobin, de-oxyhemoglobin, carboxyhemoglobin, and methemoglobin. Clin Chem 1991;37:1633-8.

40. Kollias N. The spectroscopy of human melanin pigmentation. In: Melanin: Its Role in Human Photoprotection. Overland Park, KS: Valdenmar, 1995:31-8.

41. Vreman HJ, Wong RJ, Murdock JR, Stevenson DK. Standardized bench method for evaluating the efficacy of phototherapy devices. Acta Paediatr 2008;97:308-16.

42. Vreman HJ, Wong RJ, Stevenson DK. Phototherapy: current methods and future directions. Semin Perinatol 2004;28:326-33.

43. Vreman HJ, Wong RJ, Stevenson DK, et al. Light-emitting diodes: a novel light source for phototherapy. Pediatr Res 1998;44:804-9. 